\title{
La fragilidad de las personas mayores de Gipuzkoa
}

\section{Bakarne Etxeberria Erauskin}

Programa de Atención a la Fragilidad, Fundación Hurkoa, y Departamento de Trabajo Social y Sociología, Universidad de Deusto betxeberria@hurkoa.eus

\section{Laura Gallo Rodríguez}

Ikei, Research \& Consultancy

Socióloga, consultora especializada en política social

Igallo@ikei.es

Artikulu honetan, Gipuzkoan bakarrik bizi diren adineko pertsonen kalteberatasunaren prebalentziari buruzko azterketaren emaitza nagusiak aurkezten dira. Azterketa hori Adinberriren estrategiaren baitan egin da. Ikerketari esker, hauskortasun-egoeretan eragina duten zazpi faktore identifikatu ahal izan dira eta, aldi berean, bakarrik bizi diren adinekoen arrisku-egoerak edo gizarte-kalteberatasuna zehatzago aztertu ahal izan da. Emaitzek adinekoen kalteberatasun- eta bakardade-egoeretara gerturatzeko aukera baliatu da eta, halaber, hauskortasun-egoeren ezaugarriak identifikatzeko aukera eman; horrela, aztertutako errealitatearen beharrizan instrumentalei, sozialei eta komunitarioei erantzuteko aukerak nabarmentzen dira.

\section{GAKO-HITZAK:}

Adinekoak, adinekoen arreta, bakardadea, gizartekalteberatasuna.
En este artículo se presentan los principales resultados del estudio sobre la prevalencia de la fragilidad de las personas mayores que viven solas en Gipuzkoa, realizado en el marco de la estrategia de Adinberri. La investigación ha permitido identificar siete factores que inciden en las situaciones de fragilidad, permitiendo a su vez un análisis más exhaustivo de las situaciones de riesgo o niveles de fragilidad entre las personas mayores que viven solas. Los resultados posibilitan una aproximación a los escenarios de vulnerabilidad y soledad de las personas mayores y, además, permiten identificar las características de las situaciones de fragilidad, pudiendo de esta manera responder y cubrir las necesidades tanto instrumentales como sociales y comunitarias de la realidad estudiada.

\section{Palabras Clave:}

Personas mayores, atención a las personas mayores, soledad, fragilidad. 


\section{Introducción}

La fundación Hurkoa, a lo largo de su historia, ha mostrado sensibilidad, preocupación e interés por la atención a las personas mayores. Este ha sido uno de los pilares fundamentales de la entidad desde su creación en 1978, momento en el que emerge una constatación del envejecimiento progresivo de la población guipuzcoana, que conlleva un mayor número de personas mayores en situación de dependencia, soledad y aislamiento, además de, en muchos casos, un contexto de precariedad económica. A lo largo de los años, las iniciativas, servicios y programas que se han puesto en marcha para dar respuesta a las necesidades que provienen de estas situaciones han sido diversas. No obstante, es en 2010, iniciado un proceso de reflexión sobre la estrategia de la entidad, cuando se incluye la necesidad de potenciar la atención a personas en situación de riesgo o fragilidad dentro de las líneas de acción prioritarias planificadas para los próximos años.

En esta misma línea, para finales de 2014 se constata la necesidad de continuar trabajando para mejorar las condiciones de vida de las personas mayores en situación de fragilidad, priorizando las labores de apoyo y acompañamiento orientadas al mantenimiento de su autonomía el mayor tiempo posible. Más recientemente, el Plan Estratégico 2015-2018 ahonda en esta línea de trabajo y se propone avanzar en la detección de las necesidades, dificultades y carencias de las personas que se encuentran en situación de fragilidad.

De esta manera, y con el fin de generar un diagnóstico inicial que posibilite la generación de conocimiento desde el saber de agentes clave, en 2018 se procede al diseño y posterior ejecución de un trabajo de investigación, con el apoyo de la Dirección de Empleo y Políticas Sociales del Gobierno Vasco, cuyos resultados quedan recogidos en el Informe del Proyecto de Fragilidad. Dicho estudio, de corte cualitativo, cuenta con la participación de profesionales del ámbito social y sanitario y con representantes del colectivo de personas mayores, quienes participaron en una serie de grupos focales celebrados en el marco del diseño de investigación planteado.

Después de esta primera fase de estudio-diagnóstico y su posterior publicación, Hurkoa pretende profundizar y avanzar en el análisis de este asunto con el propósito, ahora, de conocer la prevalencia de la fragilidad entre las personas mayores de sesenta y cinco años que viven solas en Gipuzkoa.

En 2018 la entidad, junto con la consultora IKEI, valiéndose de la convocatoria Adinberri -estrategia innovadora para el abordaje del envejecimiento en Gipuzkoa, promovida por la Diputación Foral-se embarca en una nueva fase de estudio, realizando en esta ocasión una encuesta entre personas mayores de sesenta y cinco años que viven solas en sus domicilios (Fundación Hurkoa, 2019). Dicha investigación se circunscribe a tres municipios guipuzcoanos con características distintas (Arrasate, Azkoitia y Pasaia), cuyos resultados agregados han permitido realizar una estimación aproximativa de las situaciones de fragilidad a nivel del conjunto del territorio de Gipuzkoa.

Estos resultados, que constituyen el contenido esencial del presente artículo y cuyas principales conclusiones serán expuestas en líneas siguientes, cuentan además con una fundamentación teórica previa del término "fragilidad" que ayudará a caracterizar el concepto, diferenciándolo y ajustándolo a la realidad a la que concierne.

A lo largo del tiempo, las distintas definiciones de fragilidad (Jáuregui y Rubin, 2012) han incorporado aspectos físicos, cognitivos y psicosociales. El debate sobre si las situaciones de fragilidad deben ser descritas en términos puramente biomédicos o deben incluirse también factores psicosociales está superado en la actualidad. En el trabajo realizado por Hurkoa se recoge que las personas mayores en situación de fragilidad son aquellas que "sin necesitar una incapacitación, precisan de orientación, asesoramiento y acompañamiento en la gestión del día a día y en la planificación de la última etapa de su vida conforme a su criterio, deseos y valores" (Hurkoa, 2018: 32).

De esta manera, las situaciones de fragilidad que se analizan en este estudio son las que afectan a aquellas personas mayores que viven solas en su domicilio y, aun manteniendo capacidad cognitiva para tomar decisiones y decidir sobre su vida, no cuentan con una red de apoyo o esta no es lo suficientemente efectiva, incidiendo este hecho en el desarrollo de su vida social y comunitaria. Se entiende, por tanto, que esta ausencia o debilidad de vínculos afecta negativamente al mantenimiento de las condiciones para una vida digna y el desarrollo del proyecto de vida personal, en tanto en cuanto no contar con el apoyo de terceros, o de personas significativas que les acompañen en la satisfacción de las necesidades, dificulta el vínculo con la comunidad y su entorno. La pérdida de autonomía, el deterioro y el aislamiento social, aceleran asimismo un proceso de debilitamiento que puede generar la invisibilización de la persona mayor frágil. Es esta definición conceptual la que ha guiado el desarrollo de la investigación presentada a continuación.

\section{Presentación de los resultados del estudio de la fragilidad de las personas mayores de Gipuzkoa}

Como se acaba de reseñar, el objetivo de este estudio ha sido conocer la situación real en la que se encuentra la población mayor de sesenta y cinco años que vive sola, analizar el conocimiento que tienen de los recursos de atención y anticipar sus necesidades 
futuras. Algo considerado especialmente importante por las entidades impulsoras del estudio en un contexto de progresivo envejecimiento, con un $22 \%$ de la población guipuzcoana que supera los sesenta y cinco años de edad y un $11 \%$ mayor de setenta y cinco.

El universo objeto de estudio ha sido la población de sesenta y cinco años o más que vive sola en su domicilio. Los datos de referencia utilizados han sido la Encuesta Demográfica de Euskadi publicada en 2016, la cual indicaba que en Gipuzkoa el $33 \%$ de las personas de sesenta y cinco años o más viven solas, lo que en cifras absolutas implica que 35.300 personas de sesenta y cinco años o más forman un hogar unipersonal en el territorio de Gipuzkoa.

Para la localización de las personas mayores se ha contado con la colaboración de los tres ayuntamientos participantes en el estudio y se ha remitido por correo ordinario una nota informativa del estudio a una muestra de personas mayores empadronadas solas en su domicilio. Posteriormente se ha completado el cuestionario por teléfono o de manera presencial. La metodología aplicada no está exenta de limitaciones, dado que la participación en el estudio era voluntaria y la propia situación de fragilidad de las personas mayores puede conllevar al rechazo a participar en este tipo de procesos.

La encuesta se ha realizado a una muestra representativa de 365 personas, de las cuales el $79 \%$ eran mujeres y el $21 \%$ hombres que vivían solos, lo que ha permitido obtener unos resultados generales con un nivel de confianza del $95,5 \%$ y un margen de error de $\pm 4 \%$.

Se diseñó un cuestionario fácil y manejable para personas mayores de sesenta y cinco años, abordando diferentes temas que permiten profundizar en las diferentes necesidades y situaciones reales en las que se encuentran éstas personas mayores. El cuestionario contenía una batería amplia de preguntas (cuarenta y ocho preguntas) que trataban de dar respuesta a las siguientes cuestiones:

- Características sociodemográficas de la persona entrevistada.

- Valoración de la salud.

- Valoración de la capacidad para realizar las actividades de la vida diaria.

- Valoración de la situación relacional.

- Valoración del bienestar emocional.

- Valoración del bienestar material: vivienda y situación económica.

- Conocimiento y relación con los servicios sociales.

- Conocimiento de la Fundación Hurkoa.

- Necesidades de atención a futuro.

Del análisis de los resultados de las encuestas realizadas se determinó la existencia de siete factores explicativos de la fragilidad entre las personas mayores que viven solas en Gipuzkoa: la salud, la capacidad para realizar las actividades de la vida diaria, la red de apoyo social, el bienestar emocional, la participación social y comunitaria, el bienestar material y la necesidad de vivienda.

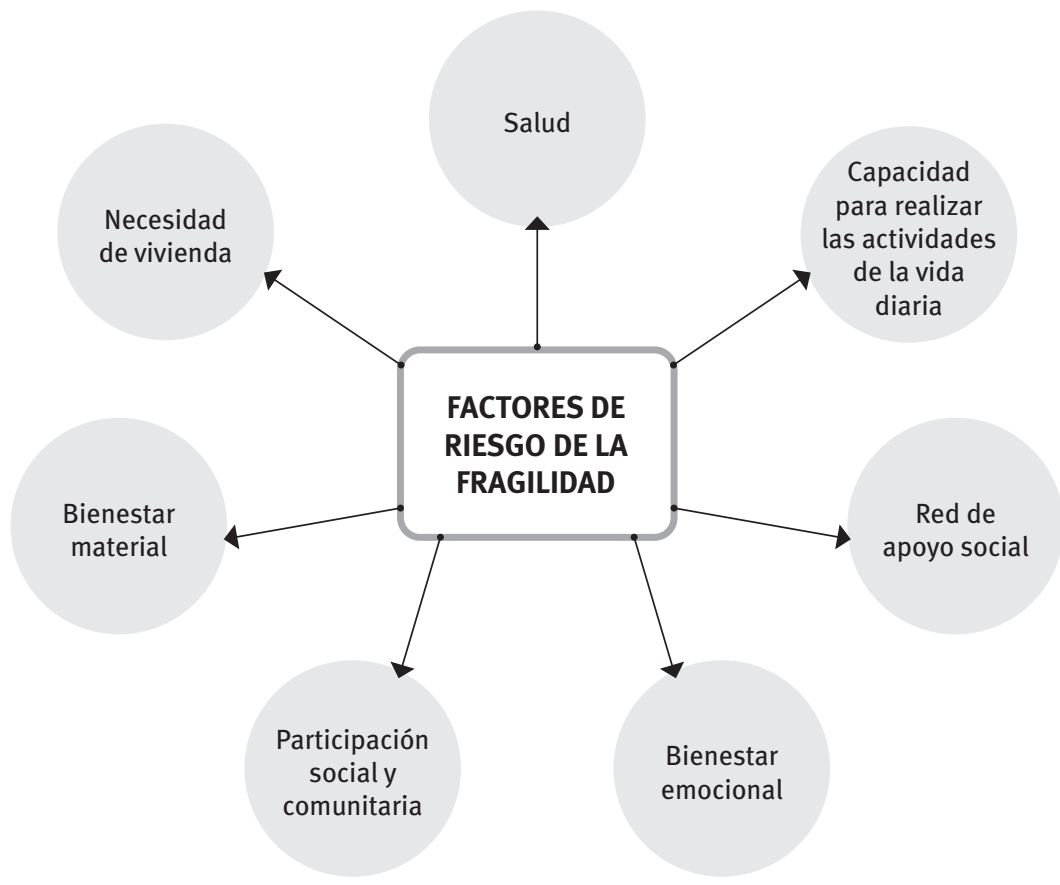

Fuente: Elaboración propia. 
De cada uno de los factores se ha recopilado información de una batería muy amplia de indicadores, de los que finalmente se seleccionaron los treinta y dos más relevantes para medir la fragilidad de las personas mayores, pudiendo hacer así una graduación de la fragilidad de cada persona mayor entrevistada. Para cada una de las dimensiones de la fragilidad se han identificado preguntas clave que son consideradas indicadores de una situación de fragilidad.

- Factor 1: salud (cinco indicadores).
- Factor 2: capacidad para realizar las actividades de la vida diaria (siete indicadores).

- Factor 3: red de apoyo social (cuatro indicadores).

- Factor 4: participación social y comunitaria (tres indicadores).

- Factor 5: bienestar emocional (cuatro indicadores).

- Factor 6: bienestar material (cinco indicadores).

- Factor 7: necesidad de vivienda (cuatro indicadores).

En la Tabla 1 se detallan los indicadores que fueron seleccionados.

Tabla 1. Factores e indicadores de fragilidad

\begin{tabular}{|c|c|c|}
\hline \multirow{2}{*}{ Factor } & \multicolumn{2}{|l|}{ INDICADOR DE FRAGILIDAD } \\
\hline & Considera que su estado de salud en general es.... & Muy malo/Malo \\
\hline \multirow{4}{*}{ 1. Salud } & ¿Ha estado hospitalizado? & Sí, en más de una ocasión \\
\hline & ¿Tiene con frecuencia problemas de salud que le impiden valerse por sí mismo? & Sí \\
\hline & ¿Hay más de dos días a la semana que no come caliente? & Sí \\
\hline & $\begin{array}{l}\text { En su opinión, ¿tiene dificultades para organizar y gestionar los medicamentos } \\
\text { que debe tomar? }\end{array}$ & Sí, alguna dificultad/ Sí, bastante dificultad \\
\hline \multirow{7}{*}{$\begin{array}{l}\text { 2. Capacidad } \\
\text { para } \\
\text { realizar las } \\
\text { actividades } \\
\text { de la vida } \\
\text { diaria }\end{array}$} & $\begin{array}{l}\text { En general, ¿cómo califica su capacidad para realizar las actividades y labores } \\
\text { habituales de la vida diaria? }\end{array}$ & Muy mala/Mala \\
\hline & Grado de autonomía: lavarse (bañarse) & Con dificultad (dependiente) \\
\hline & Grado de autonomía: vestirse y desvestirse & Con dificultad (dependiente) \\
\hline & Grado de autonomía: moverse dentro de casa (50 metros) & Con dificultad (dependiente) \\
\hline & Grado de autonomía: moverse fuera de casa (caminar 400 metros sin fatigarse) & Con dificultad (dependiente) \\
\hline & Grado de autonomía: tareas domésticas (limpieza, comida). & Con dificultad (dependiente) \\
\hline & Grado de autonomía: realizar gestiones económicas o legales. & Con dificultad (dependiente) \\
\hline \multirow{4}{*}{$\begin{array}{l}\text { 3. Red de } \\
\text { apoyo social }\end{array}$} & ¿Dispone de apoyo familiar suficiente? & No \\
\hline & ¿Necesita que le ayude alguien a menudo? & Sí \\
\hline & ¿Tiene relación con amigos/as? & $\begin{array}{l}\text { No tiene ese vínculo/Tiene esa red social, } \\
\text { pero no se relaciona con ellos }\end{array}$ \\
\hline & ¿Conoce los servicios sociales? & No \\
\hline \multirow{3}{*}{$\begin{array}{l}4 . \\
\text { Participación } \\
\text { social y } \\
\text { comunitaria }\end{array}$} & Frecuencia para salir de casa & Mensualmente o menos \\
\hline & $\begin{array}{l}\text { Frecuencia para realizar actividades de participación social (hacer recados, ir al } \\
\text { bar o a la cafetería, ir al hogar del jubilado). }\end{array}$ & No voy nunca \\
\hline & $\begin{array}{l}\text { Frecuencia para realizar actividades de participación comunitaria (salir a } \\
\text { pasear, hacer deporte, ir a actos deportivos o culturales, cursos de formación, } \\
\text { vacaciones o voluntariado). }\end{array}$ & No voy nunca \\
\hline \multirow{4}{*}{$\begin{array}{l}\text { 5. Bienestar } \\
\text { emocional }\end{array}$} & Percepción de soledad. & Sí, a menudo \\
\hline & Percepción de inseguridad en la vivienda (miedo a que le roben, etc.). & Sí, a menudo \\
\hline & Percepción de inseguridad en el entorno (barrio). & Sí, a menudo \\
\hline & Percepción de maltrato físico o psicológico. & Sí, a menudo/Sí, alguna vez \\
\hline \multirow{5}{*}{$\begin{array}{l}\text { 6. Bienestar } \\
\text { material }\end{array}$} & $\begin{array}{l}\text { En los últimos doce meses, ¿ha tenido dificultad para afrontar los gastos } \\
\text { generales (alquiler, electricidad, gas, comida, ropa, etc.? }\end{array}$ & Mucha dificultad \\
\hline & Cuál es su nivel de ingresos mensual actual. & Menos de 700 euros al mes \\
\hline & Régimen de tenencia de la vivienda en la que reside. & $\begin{array}{l}\text { Una vivienda propia en proceso de pago/ } \\
\text { Una vivienda de alquiler }\end{array}$ \\
\hline & ¿La vivienda tiene calefacción? & $\begin{array}{l}\text { No, no tengo/Sí, tengo calefacción pero no } \\
\text { la pongo }\end{array}$ \\
\hline & Percepción de abuso económico. & Sí, a menudo/Sí, alguna vez \\
\hline \multirow{4}{*}{$\begin{array}{l}\text { 7. Necesidad } \\
\text { de vivienda }\end{array}$} & El edificio es accesible hasta el portal (sin escaleras, con rampa). & No \\
\hline & Cuenta con ascensor hasta la planta. & No \\
\hline & La vivienda es accesible por dentro (tiene el baño adaptado). & No \\
\hline & ¿Actualmente tiene necesidad de cambiar o de reformar la vivienda? & $\begin{array}{l}\text { Necesito cambiar de vivienda/Necesito } \\
\text { reformar o rehabilitar la vivienda }\end{array}$ \\
\hline
\end{tabular}

Fuente: Fundación Hurkoa 2019. 
Con los datos recogidos en cada uno de los indicadores y dimensiones de la fragilidad se realiza una clasificación de la población mayor en función de su nivel de fragilidad. Se definen cuatro niveles de fragilidad (la situación no problemática, moderada, grave y muy grave) de acuerdo con las siguientes definiciones:

- No problemática: persona que cuenta con cinco o menos indicadores de riesgo.

- Moderada: persona que tiene entre seis y diez indicadores de riesgo.

- Grave: persona que tiene entre once y catorce indicadores de riesgo.

- Muy grave: persona con quince o más indicadores de riesgo.

Tabla 2. Niveles de fragilidad

\begin{tabular}{|l|l|}
\hline Nivel de fragilidad & Indicadores \\
\hline No problemática & $0-5$ indicadores \\
\hline Moderado & $6-10$ indicadores \\
\hline Grave & $11-14$ indicadores \\
\hline Muy grave & 150 más indicadores \\
\hline
\end{tabular}

Fuente: Elaboración propia.

También se calcula para cada uno de los factores el grado de riesgo en el que se encuentran las personas mayores, estableciéndose tres niveles: las personas que se encuentran sin riesgo en ese factor, las personas en las que presentan una situación de prefragilidad, dado que cuentan con algún indicador de fragilidad en dicha dimensión, y las personas que ya están experimentando una situación de fragilidad en ese factor (más de dos indicadores de fragilidad). En la Tabla 3 se recogen los criterios establecidos:

Tabla 3. Niveles de fragilidad de cada factor

\begin{tabular}{|l|c|c|l|}
\hline \multirow{2}{*}{ Factor de fragilidad } & \multicolumn{2}{|c|}{ Nivel de fragilidad del factor } \\
\cline { 2 - 4 } & $\begin{array}{c}\text { Sin riesgo } \\
(\mathbf{0})\end{array}$ & $\begin{array}{c}\text { Prefrágil } \\
(1-2)\end{array}$ & Frágil(>2) \\
\hline 1. Salud & $\begin{array}{c}\text { Sin riesgo } \\
(0)\end{array}$ & $\begin{array}{c}\text { Pre-frágil } \\
(1-2)\end{array}$ & Frágil(>2) \\
\hline $\begin{array}{l}\text { 2. Capacidad para } \\
\text { realizar las actividades de } \\
\text { la vida diaria }\end{array}$ & $\begin{array}{c}\text { Sin riesgo } \\
(0)\end{array}$ & $\begin{array}{c}\text { Pre-frágil } \\
(1-2-3)\end{array}$ & Frágil(>3) \\
\hline $\begin{array}{l}\text { 3. Red de apoyo social } \\
\text { 4. Participación social y } \\
\text { comunitaria }\end{array}$ & $\begin{array}{c}\text { Sin riesgo } \\
(0)\end{array}$ & $\begin{array}{c}\text { Pre-frágil } \\
(1-2)\end{array}$ & Frágil(>2) \\
\hline 5. Bienestar emocional & $\begin{array}{c}\text { Sin riesgo } \\
(0)\end{array}$ & $\begin{array}{c}\text { Pre-frágil } \\
(1)\end{array}$ & Frágil(>1) \\
\hline 6. Bienestar material & $\begin{array}{c}\text { Sin riesgo } \\
(0)\end{array}$ & $\begin{array}{c}\text { Pre-frágil } \\
(1-2)\end{array}$ & Frágil(>2) \\
\hline 7. Necesidad de vivienda & $\begin{array}{c}\text { Sin riesgo } \\
(0)\end{array}$ & $\begin{array}{c}\text { Pre-frágil } \\
(1-2)\end{array}$ & Frágil(>2) \\
\hline
\end{tabular}

Fuente: Elaboración propia.
A continuación se presentan los principales resultados de Gipuzkoa recogidos en el estudio publicado por la Fundación Hurkoa en 2019, La fragilidad de las personas mayores en Gipuzkoa.

\section{Valoración general de las situaciones de fragilidad en Gipuzkoa}

El estudio realizado por la Fundación Hurkoa refleja en general una situación positiva de las personas mayores que viven solas en Gipuzkoa, aunque con algunos aspectos de mejora sobre los que trabajar para frenar o retrasar el proceso que lleva a muchas personas mayores a encontrarse en situaciones de fragilidad.

El aumento de las personas que viven solas está estrechamente relacionado con la alta capacidad que las personas mayores de Gipuzkoa tienen para llevar una vida autónoma e independiente. La gran mayoría de las personas mayores que viven solas confirma que tiene una alta capacidad para desenvolverse adecuadamente tanto en las actividades y labores más básicas de la vida diaria (vestirse, comer, moverse dentro y fuera de casa, etc.), como en las instrumentales (utilizar el teléfono, hacer las tareas domésticas, gestionar el dinero, etc.). En ese sentido, casi tres de cada cuatro personas mayores de Gipuzkoa (el $73 \%$ de las personas que viven solas entrevistadas) creen que su capacidad para realizar las actividades y labores habituales de la vida diaria es buena o muy buena.

Además, estas personas mayores cuentan en general con una buena salud, un adecuado bienestar material (capacidad de pago adecuada y necesidad de vivienda cubierta) y buenos niveles de relación y de participación social y comunitaria. De hecho, siete de cada diez personas mayores de sesenta y cinco años que viven solas entrevistadas en Gipuzkoa confirman que tienen una buena calidad de vida.

Ahora bien, la investigación llevada a cabo por Hurkoa ha permitido identificar situaciones de riesgo y de fragilidad, destacando la debilitada salud de una parte de la población mayor que dificulta la realización de las actividades más básicas de la vida diaria, la percepción de la soledad que limita su participación social en la comunidad, o la residencia en edificios y viviendas con necesidades de adecuación de la accesibilidad para facilitar los movimientos de las personas. 


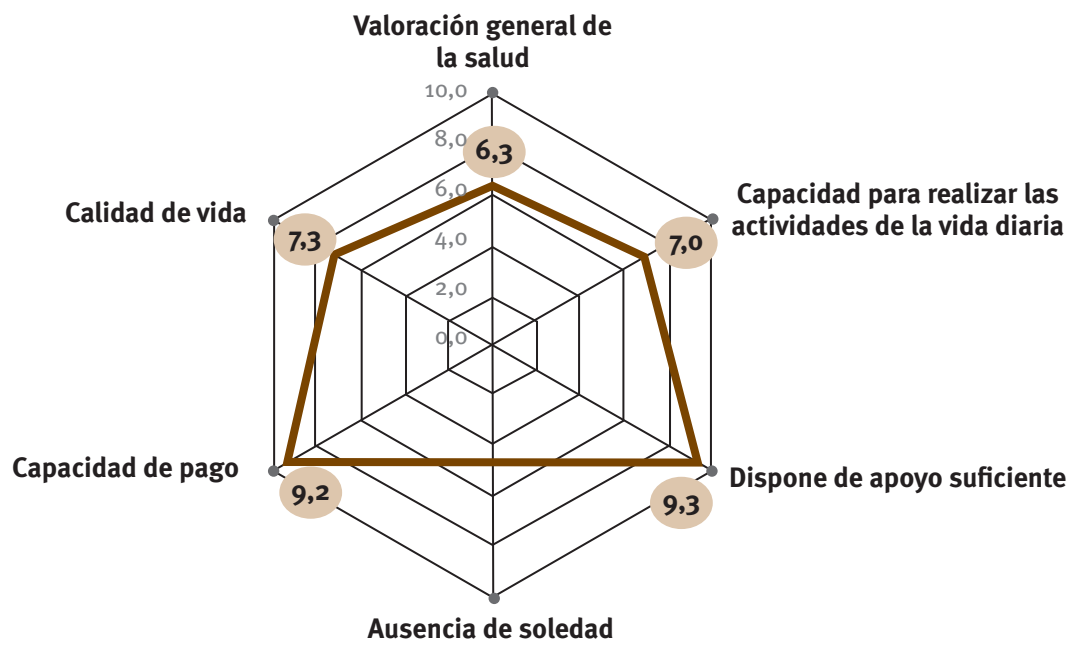

Índice 0-10

Ausencia de soledad

Fuente: Elaboración propia.

Tabla 4. Principales resultados de la encuesta sobre la fragilidad realizada a población de 65 años que vive sola de Gipuzkoa

\begin{tabular}{|c|c|c|}
\hline \multicolumn{2}{|l|}{ Indicador } & \multirow{2}{*}{$\begin{array}{c}\begin{array}{r}\text { Estimación } \\
\text { Gipuzkoa* }\end{array} \\
6,3\end{array}$} \\
\hline \multirow{5}{*}{$\begin{array}{l}\text { Salud y capacidad } \\
\text { para realizar las } \\
\text { actividades de la vida } \\
\text { diaria }\end{array}$} & Valoración general de la salud (índice o-10) & \\
\hline & - Dificultades para organizar y gestionar medicamentos (\% afirmativo) & $2 \%$ \\
\hline & - Problemas de salud que le impiden valerse por sí mismo (\% afirmativo) & $9,6 \%$ \\
\hline & - Le impide la salud salir a la calle (\% afirmativo) & $9,3 \%$ \\
\hline & Capacidad para realizar las actividades de la vida diaria (índice $0-10$ ) & 7 \\
\hline \multirow{5}{*}{$\begin{array}{l}\text { Situación relacional y } \\
\text { participación social y } \\
\text { comunitaria }\end{array}$} & Relación con la familia próxima diaria (\% afirmativo) & $82,1 \%$ \\
\hline & No tiene relación o no tiene familia próxima (\% afirmativo) & $6,6 \%$ \\
\hline & No dispone de apoyo suficiente (\% afirmativo) & $5,8 \%$ \\
\hline & Se encuentra sin nadie a quien acudir si precisa ayuda (\% afirmativo) & $2,5 \%$ \\
\hline & No sale de casa (\% afirmativo) & $0,8 \%$ \\
\hline \multirow{4}{*}{ Bienestar emocional } & Percepción de soledad (\% a menudo) & $17,5 \%$ \\
\hline & Percepción de tristeza (\% a menudo) & $21,1 \%$ \\
\hline & Inseguridad en la vivienda (\% a menudo) & $5,3 \%$ \\
\hline & Dificultades o ansiedad para realizar algunas gestiones diarias (\% a menudo) & $2,2 \%$ \\
\hline \multirow{7}{*}{$\begin{array}{l}\text { Bienestar material: } \\
\text { situación de la } \\
\text { vivienda y nivel de } \\
\text { ingresos }\end{array}$} & Vivienda de alquiler (\%) & $3,6 \%$ \\
\hline & Necesidad de cambiar o de rehabilitar (\%) & $7,3 \%$ \\
\hline & El edificio no es accesible (\%) & $42,8 \%$ \\
\hline & No se dispone de ascensor hasta planta (\%) & $34 \%$ \\
\hline & Dificultad para afrontar los gastos generales (\% mucha o alguna dificultad) & $12,6 \%$ \\
\hline & No cuenta con calefacción o no la pone (\%) & $17,1 \%$ \\
\hline & Ingresos medios mensuales (euros) & $1.005,6$ \\
\hline $\begin{array}{l}\text { Valoración general de } \\
\text { la calidad de vida }\end{array}$ & Valoración media de la calidad de vida (índice o-10) & 7,3 \\
\hline
\end{tabular}

* Estimación por extrapolación de los resultados del estudio al conjunto de la población mayor de 65 años que vive sola en Gipuzkoa. Fuente: Fundación Hurkoa, 2019. 
Tomando en consideración los 32 indicadores más relevantes de la fragilidad, el estudio ha permitido estimar que el $87 \%$ de las personas mayores de 65 años que viven solas en Gipuzkoa no presenta una situación problemática. Por su parte, el $12 \%$ de las personas mayores de Gipuzkoa que viven solas tiene un nivel de fragilidad calificado como moderado, dado que han respondido afirmativamente a entre 6 y 10 de los 32 indicadores seleccionados. Y, finalmente, se estima que un $1 \%$ de las personas mayores entrevistadas presenta un grado de fragilidad calificado como grave. En el momento de la investigación, no se han identificado personas mayores de 65 años que vivan solas y que presentasen una situación de fragilidad calificada como muy grave, aunque hay que recordar que la participación en el estudio era voluntaria, lo que planteaba por tanto dificultades para detectar situaciones altamente graves.

Tomando como referencia la cifra de 35.300 personas mayores de 65 años en Gipuzkoa que conforman un hogar unipersonal y el grado de fragilidad calculado, cabría estimar que hay unas 4.255 personas mayores de Gipuzkoa se encuentran en una situación de fragilidad moderada y otras 484 personas con una fragilidad que puede calificarse como grave (al presentar entre 11 y 14 de los 32 indicadores planteados).

Tabla 5. Grado de fragilidad de las personas mayores de 65 años que viven solas en Gipuzkoa

\begin{tabular}{|l|c|c|}
\cline { 2 - 3 } \multicolumn{1}{c|}{} & $\begin{array}{c}\text { Resultado de } \\
\text { la encuesta } \\
\text { (\% vertical) }\end{array}$ & $\begin{array}{c}\text { Estimación } \\
\text { Gipuzkoa (N) }\end{array}$ \\
\hline $\begin{array}{l}\text { No problemática } \\
\text { (o-5 indicadores) }\end{array}$ & 86,6 & 30.561 \\
\hline Moderada (6-10 indicadores) & 12,1 & 4.255 \\
\hline Grave (11-14 indicadores) & 1,4 & 484 \\
\hline $\begin{array}{l}\text { Muy grave (más de 14 } \\
\text { indicadores) }\end{array}$ & 0 & 0 \\
\hline Total & 100 & 35.300 \\
\hline
\end{tabular}

* Estimación por extrapolación de los resultados del estudio al conjunto de población mayor de 65 años que vive sola en Gipuzkoa. Fuente: Fundación Hurkoa, 2019.

Ahondando en la capacidad de reacción de las personas mayores ante posibles situaciones de fragilidad, se recoge también información sobre el
Gráfico 3. Grado de fragilidad de las personas mayores de 65 años que viven solas en Gipuzkoa

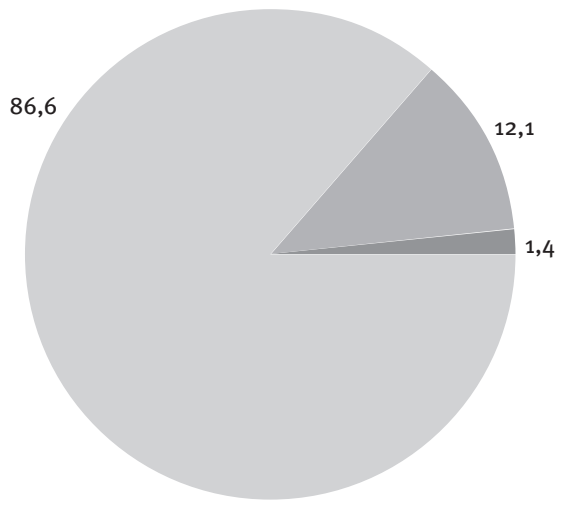

No problemática (0-5)

Moderada (6-10)

Grave (11-14)

* Estimación por extrapolación de los resultados del estudio a conjunto de población mayor de 65 años que vive sola en Gipuzkoa. Fuente: Fundación Hurkoa, 2019.

conocimiento que tienen de los servicios sociales. Del estudio se desprende que en general el nivel de conocimiento es relativamente amplio. En concreto, el $87 \%$ de las personas mayores que viven solas afirma conocer los servicios sociales, mientras que un $12 \%$ declara que no conoce los servicios sociales, un $16 \%$ entre los hombres frente a un $11 \%$ de las mujeres entrevistadas.

También se recoge la opinión de las personas mayores de Gipuzkoa sobre la necesidad en un futuro cercano de acudir a los servicios sociales. El 61,5\% de las personas mayores de 65 entrevistadas que viven solas declara que es muy probable o bastante probable que acuda en un futuro cercano. Un $65 \%$ de las mujeres y en menor medida un $49 \%$ de los hombres lo ha declarado así.

Pensando en el diseño de estrategias a futuro, la encuesta expone a las personas mayores residentes en Gipuzkoa una batería amplia de servicios de apoyo y les pide que indiquen si les parecen interesantes. En general, ocho de cada diez personas entrevistadas

Tabla 6. Conocimiento de los recursos de atención y demanda a futuro de recursos

\begin{tabular}{|c|c|c|}
\hline \multicolumn{2}{|l|}{ Indicador } & Estimación Gipuzkoa* \\
\hline \multirow{4}{*}{$\begin{array}{l}\text { Conocimiento y uso de los } \\
\text { recursos de atención }\end{array}$} & Conocimiento de los servicios sociales (\% afirmativo) & $87,1 \%$ \\
\hline & Desconocimiento de los servicios sociales (\% no conoce) & $12,3 \%$ \\
\hline & Uso de los recursos de atención de los servicios sociales en el último año (\%) & $15,1 \%$ \\
\hline & Valoración media sobre los servicios sociales (índice 0-10) & 8,8 puntos \\
\hline $\begin{array}{l}\text { Demanda a futuro de recursos } \\
\text { de atención }\end{array}$ & Necesidad de los servicios en el futuro cercano (\% afirmativo) & $61,5 \%$ \\
\hline
\end{tabular}

*Estimación por extrapolación de los resultados del estudio al conjunto de la población mayor de 65 años que vive sola en Gipuzkoa.

Fuente: Fundación Hurkoa, 2019. 
indican que por el momento no están interesadas en recibir nuevos recursos de apoyo. Ahora bien, poniendo la mirada en los niveles de interés positivos, hay que destacar que el $20 \%$ de las personas que viven solas podrían estar interesadas en recibir apoyos para realizar las tareas básicas, como son las tareas personales y domésticas. Y aproximadamente un $5 \%$ considera interesante contar con apoyo para realizar actividades de la vida diaria fuera del hogar (hacer compras, ir a la peluquería, actividades de ocio, etc.) o apoyo psicológico o psicosocial.

\section{Los siete factores de la fragilidad}

Como ya se indicaba con anterioridad, el estudio ha permitido profundizar en los siete factores de fragilidad de las personas mayores y la investigación ha posibilitado identificar situaciones de riesgo o de vulnerabilidad entre las personas mayores que viven solas en cada uno de los factores de la fragilidad estudiados. Se describe a continuación una síntesis de la situación detectada en cada uno de los factores.

\section{Factor 1. Salud}

Comenzando por el factor de la salud, la encuesta solicita a la población mayor de 65 años que vive sola que califique su estado de salud en general y si cree que tiene con frecuencia problemas de salud que le impiden valerse por sí misma. También se analizan otros indicadores, como haber estado hospitalizado en los últimos doce meses en una o varias ocasiones, no comer caliente más de dos días a la semana 0 tener dificultades para organizar y gestionar los medicamentos que debe tomar.

La investigación permite concluir que en Gipuzkoa una parte muy relevante de las personas mayores que viven solas considera que cuenta con un estado de salud bueno, con una valoración media de 6,3 puntos sobre 10 . Sin embargo, un $5 \%$ califica su estado de salud actual como malo o muy malo. Además, un $10 \%$ de las personas que viven solas reconocen que tienen problemas de salud que les impiden valerse por sí mismas y aproximadamente un $9 \%$ declara que no puede salir a la calle. Un resultado relevante apunta a que entre las personas mayores que viven solas se detecta un $7 \%$ que afirma que hay más de dos días a la semana en los que no come caliente.

Son muy pocas las personas que indican que tienen dificultades para gestionar y organizar los medicamentos, ya que solo un $2 \%$ de las personas mayores de 65 años de Gipuzkoa que viven solas reconoce tener esa dificultad. Ahora bien, hay que precisar que aproximadamente un $10 \%$ señala que no tiene dificultad porque recibe ayuda.

En el factor de salud se ha calificado como persona frágil aquella que presenta más de dos de las siguientes situaciones:
- Considera que su estado de salud en general es malo o muy malo.

- Ha estado hospitalizada en más de una ocasión en el último año.

- Tiene con frecuencia problemas de salud que le impiden valerse por sí misma

- Hay más de dos días a la semana que no come caliente.

- Tiene dificultades para organizar y gestionar los medicamentos que debe tomar.

Del total de las personas mayores de 65 años que viven solas resulta que tienen una situación de fragilidad por motivos de salud el 0,8\%. En términos poblacionales, cabe estimar que hay 290 personas mayores que viven solas y que tiene fragilidad por motivos de salud en Gipuzkoa. Por otro lado, del estudio se desprende que un $19 \%$ de las personas mayores viven actualmente en una situación de prefragilidad (aproximadamente 6.600 personas), ya que han reconocido una o dos situaciones de las mencionadas anteriormente.

Tabla 7. Salud como factor de riesgo de la fragilidad de las personas mayores de 65 años que viven solas en Gipuzkoa

\begin{tabular}{|l|c|c|c|}
\hline \multirow{2}{*}{ Salud } & \multicolumn{2}{|c|}{ Resultados de la encuesta } & $\begin{array}{c}\text { Estimación } \\
\text { Gipuzkoa* }\end{array}$ \\
\cline { 2 - 4 } & $\mathbf{N}$ & $\%$ & $\mathbf{N}$ \\
\hline Sin riesgo & 294 & 80,5 & 28.433 \\
\hline Prefrágil & 68 & 18,6 & 6.576 \\
\hline Frágil & 3 & 0,8 & 290 \\
\hline Total & 365 & 100 & 35.300 \\
\hline
\end{tabular}

* Estimación por extrapolación de los resultados del estudio al conjunto de población mayor de 65 años que vive sola en Gipuzkoa. Fuente: Fundación Hurkoa, 2019.

\section{Factor 2. Capacidad para realizar las actividades} de la vida diaria

El segundo factor estudiado es la capacidad para realizar las actividades y las labores habituales de la vida diaria. Para ello se le pide a la persona que ofrezca una valoración personal sobre su grado de autonomía en diferentes actividades, como lavarse (bañarse), vestirse y desvestirse, moverse dentro de casa (50 metros) o fuera de casa (caminar 400 metros sin fatigarse). Igualmente se estudia su capacidad para realizar otras actividades calificadas como instrumentales, como son utilizar el teléfono, ir de compras, realizar las tareas domésticas (limpieza, comida) o gestiones económicas o legales.

En este sentido, se estima que el $73 \%$ de las personas que viven solas cree que su capacidad para realizar las actividades y labores habituales de la vida diaria es buena o muy buena, frente al 5,5\% que considera su capacidad como mala o muy mala.

La falta de capacidad para realizar las actividades de la vida diaria más básicas y las instrumentales se acrecienta entre las personas de 85 años y 
más. No obstante, es interesante constatar que la capacidad más limitada se señala en la mayoría de los casos por personas que ya son usuarias de los servicios sociales. Del estudio se desprende que las mujeres manifiestan una capacidad menor para realizar gestiones y compras que los hombres, mientras que los hombres sufren más dificultades para desempeñar tareas domésticas. La encuesta ha identificado un colectivo especialmente frágil, que afecta al 1,4\% de las personas mayores que viven solas, que reconoce tener dificultades para tomar decisiones.

En el factor de la capacidad para realizar las actividades de la vida diaria se califica como persona frágil aquella persona que presenta más de tres de las siguientes situaciones:

- Califica su capacidad para realizar las actividades y labores habituales de la vida diaria como mala o muy mala.

- Declara dificultad para lavarse (bañarse).

- Declara dificultad para vestirse y desvestirse.

- Declara dificultad para moverse dentro de casa (50 metros).

- Declara dificultad para moverse fuera de casa (caminar 400 metros sin fatigarse).

- Declara dificultad para realizar tareas domésticas (limpieza, comida).

- Declara dificultad para realizar gestiones económicas o legales.

Con arreglo a estos criterios, resulta que tienen una situación de fragilidad por tener dificultades para realizar las actividades de la vida diaria el 1,9\% del total de las personas mayores de 65 años que viven solas. En términos poblacionales y teniendo en cuenta los resultados del estudio, cabe estimar que en Gipuzkoa residen 677 personas mayores que viven solas con dificultades para realizar las actividades de la vida diaria. Un $18 \%$ de las personas mayores viven actualmente en una situación de prefragilidad (aproximadamente 6.300 personas en Gipuzkoa), personas que han reconocido tener dificultades entre una y tres actividades de la vida diaria mencionadas anteriormente.

Tabla 8. Capacidad para realizar las actividades de la vida diaria como factor de riesgo de la fragilidad de las personas mayores de 65 años que viven solas en Gipuzkoa

\begin{tabular}{|l|c|c|c|}
\hline \multirow{2}{*}{$\begin{array}{l}\text { Capacidad para } \\
\text { realizar las } \\
\text { actividades de } \\
\text { la vida diaria }\end{array}$} & \multicolumn{2}{|c|}{ Resultados de la Encuesta } & $\begin{array}{c}\text { Estimación } \\
\text { Gipuzkoa* }\end{array}$ \\
\cline { 2 - 4 } & N & $\%$ & N \\
\hline Sin riesgo & 293 & 80,3 & 28.337 \\
\hline Pre-frágil & 65 & 17,8 & 6.286 \\
\hline Frágil & 7 & 1,9 & 677 \\
\hline Total & 365 & 100,0 & 35.300 \\
\hline
\end{tabular}

* Estimación por extrapolación de los resultados del estudio al conjunto de población mayor de 65 años que vive sola en Gipuzkoa. Fuente: La fragilidad de las personas mayores en Gipuzkoa. Fundación Hurkoa, 2019.

\section{Factor 3. Red de apoyo social}

El tercer factor se refiere a la red social y los apoyos de otras personas, factor clave para mitigar las situaciones de soledad y evitar la fragilidad. La encuesta recoge información sobre la relación que tienen las personas mayores que viven solas con su familia próxima (hijo/os, hija/as, hermanos/as, nietos/as), con la familia extensa (tíos/tías, primos/as, sobrinos/as), con las amistades y con el vecindario.

La fragilidad es un aspecto muy vinculado a la falta de red social de apoyo de las personas mayores en su día a día. La gran mayoría de las personas mayores de 65 años encuestadas declara tener unas relaciones sociales frecuentes, sobre todo con la familia más próxima: ocho de cada diez personas afirman tener relación a diario con hijos/as, hermanos/as o nietos/ as. Ahora bien, un $7 \%$ de las personas que viven solas indica que no tienen relación o no tienen familia próxima y un $6 \%$ expone que no dispone de apoyo familiar suficiente. Hay, además, un 2,5\% que no puede pedir ayuda a nadie si la necesita.

En la dimensión de la red de apoyo social se califica como persona frágil aquella persona que presenta más de dos de las siguientes situaciones:

- No dispone de apoyo familiar suficiente.

- Necesita que le ayude alguien a menudo.

- No tiene relación con las amistades.

- No conoce los servicios sociales.

De acuerdo con este criterio, el 0,3\% de las personas mayores de 65 años que viven solas de Gipuzkoa se encuentra en una situación de fragilidad por falta de red de apoyo social, por lo que en cifras absolutas podríamos estar hablando de aproximadamente 100 personas.

Ahora bien, es importante destacar que un $37 \%$ de las personas mayores que viven solas se encuentran en una situación de prefragilidad por tener una red de apoyo social débil (reconocen vivir una o dos situaciones de las recogidas anteriormente). En términos poblacionales, se estima que algo más de 13.000 personas mayores presentan una situación de prefragilidad por falta de una red de apoyo social.

Tabla 9. Red de apoyo social como factores de riesgo de la fragilidad de las personas mayores de 65 años que viven solas en Gipuzkoa

\begin{tabular}{|l|c|c|c|}
\hline \multirow{2}{*}{$\begin{array}{l}\text { Red de Apoyo } \\
\text { Social }\end{array}$} & \multicolumn{2}{|c|}{ Resultados de la encuesta } & $\begin{array}{c}\text { Estimación } \\
\text { Gipuzkoa* }\end{array}$ \\
\cline { 2 - 4 } & $\mathbf{N}$ & $\%$ & N \\
\hline Sin riesgo & 229 & $62, \%$ & 22.147 \\
\hline Prefrágil & 135 & 37,0 & 13.056 \\
\hline Frágil & 1 & 0,3 & 97 \\
\hline Total & $\mathbf{3 6 5}$ & $\mathbf{1 0 0 , 0}$ & $\mathbf{3 5 . 3 0 0}$ \\
\hline
\end{tabular}

* Estimación por extrapolación de los resultados del estudio al conjunto de población mayor de 65 años que vive sola en Gipuzkoa. Fuente: Fundación Hurkoa, 2019. 
El cuarto factor estudiado alude a la participación social y comunitaria de las personas mayores que viven solas. Se realiza una consulta sobre la frecuencia con la que sale de casa, hace recados (compras, banco, farmacia, etc.), sale a pasear o acude a un bar o a una cafetería o al hogar del jubilado. También se analizan actividades como la práctica deportiva, la asistencia a cursos de formación o la realización de actividades de voluntariado.

El $84,5 \%$ de las personas mayores que viven solas sale de casa todos los días, un 10,5\% lo hace una o varias veces a la semana y un $8 \%$ sale con menor frecuencia ( $3,3 \%$ una o varias veces al mes y $1,6 \%$ no sale o sale tan solo una o varias veces al año).

La realización de recados como hacer las compras, ir al banco o ir a la farmacia se lleva a cabo todos los días o varias veces a la semana y hay un porcentaje, aunque menor, que las hace una vez al mes. Son muchas las personas mayores que viven solas que salen a pasear todos los días (70\%). Solo el $22 \%$ practica algún deporte y también es reducido el número de personas que acuden a actos deportivos, religiosos o culturales.

Según el factor de la participación social y comunitaria se califica como persona frágil aquella que presenta más de una de las siguientes situaciones:

- En el último año no ha salido de casa.

- No realiza ninguna de las siguientes actividades de participación social: hacer recados, ir al bar o a la cafetería, ir al hogar del jubilado.

- No realiza actividades de participación comunitaria: salir a pasear, hacer deporte, ir a actos deportivos o culturales, cursos de formación, vacaciones o voluntariado.

El resultado es que el 1,9\% de las personas mayores de 65 años que viven solas de Gipuzkoa se encuentra en una situación de fragilidad por su falta de participación social y comunitaria. En términos poblacionales, se estima que hay 677 personas en Gipuzkoa que podrían estar en esta situación.

Si una persona indica alguna de las tres opciones planteadas, se la califica como prefrágil en el ámbito de la participación social y comunitaria. Esta circunstancia afectaría a un 3,6 \% de la población mayor que vive sola en Gipuzkoa (aproximadamente 1.300 personas).
Tabla 10. Participación social y comunitaria como factor de riesgo de la fragilidad de las personas mayores de 65 años que viven solas en Gipuzkoa

\begin{tabular}{|l|c|c|c|}
\hline \multirow{2}{*}{$\begin{array}{l}\text { Participación } \\
\text { Social y } \\
\text { Comunitaria }\end{array}$} & \multicolumn{2}{|c|}{ Resultados de la encuesta } & $\begin{array}{c}\text { Estimación } \\
\text { Gipuzkoa* }\end{array}$ \\
\cline { 2 - 4 } & $\mathbf{N}$ & $\%$ & $\mathbf{N}$ \\
\hline Sin riesgo & 345 & 94,5 & 33.366 \\
\hline Prefrágil & 13 & 3,6 & 1.257 \\
\hline Frágil & 7 & 1,9 & 677 \\
\hline Total & 365 & $\mathbf{1 0 0 , 0}$ & $\mathbf{3 5 . 3 0 0}$ \\
\hline
\end{tabular}

* Estimación por extrapolación de los resultados del estudio al conjunto de población mayor de 65 años que vive sola en Gipuzkoa. Fuente: Fundación Hurkoa, 2019.

\section{Factor 5. Bienestar emocional}

El quinto factor corresponde al bienestar emocional. La encuesta indaga sobre la percepción que las personas mayores que viven solas tienen sobre la soledad, la tristeza, la vivencia del miedo a olvidarse de ciertas cosas (fuego, gas, calefacción, grifo.., etc.), la percepción de inseguridad en la vivienda (miedo a que le roben, etc.) o en el entorno (barrio) o la experimentación de dificultades o ansiedad para realizar algunas gestiones diarias (banco, seguros, abogados, etc.) o vivencias de rechazo o discriminación (por la edad) e incluso de abuso económico, maltrato físico o psicológico.

De acuerdo con los datos de la encuesta, la percepción de soledad y de tristeza es bastante acusada entre las personas mayores que viven solas en Gipuzkoa, estimándose que un $17,5 \%$ de las personas que viven solas reconoce que siente a menudo la soledad y un $21 \%$ la tristeza. Cerca de un $2 \%$ asegura haber sufrido en alguna ocasión abuso económico o maltrato físico o psicológico.

En la dimensión de bienestar emocional se califica como persona frágil aquella persona que presenta más de dos de las siguientes situaciones:

- Percepción frecuente de soledad.

- Percepción frecuente de inseguridad en la vivienda (miedo a que le roben, etc.).

- Percepción frecuente de inseguridad en el entorno (barrio).

- Percepción frecuente de maltrato físico o psicológico.

La falta de bienestar emocional es el factor de fragilidad reconocido por el 0,3\% de las personas mayores de 65 años que viven solas de Gipuzkoa. En cifras absolutas, el número estimado es reducido, 97 personas.

Ahora bien, el $20 \%$ de las personas mayores que viven solas plantea una situación de prefragilidad, ya que han indicado una o dos situaciones de fragilidad del bienestar emocional. Se estima, por tanto, que unas 7.000 personas mayores de Gipuzkoa presentan una situación de prefragilidad por debilidad en el bienestar emocional. 
Tabla 11. Bienestar emocional como factor de riesgo de la fragilidad de las personas mayores de 65 años que viven solas en Gipuzkoa

\begin{tabular}{|l|c|c|c|}
\hline \multirow{2}{*}{$\begin{array}{l}\text { Bienestar } \\
\text { Emocional }\end{array}$} & \multicolumn{2}{|c|}{ Resultados de la encuesta } & $\begin{array}{c}\text { Estimación } \\
\text { Gipuzkoa* }\end{array}$ \\
\cline { 2 - 4 } & $\mathbf{N}$ & $\%$ & $\mathbf{N}$ \\
\hline Sin riesgo & 291 & 79,7 & 28.143 \\
\hline Prefrágil & 73 & 20,0 & 7.060 \\
\hline Frágil & 1 & 0,3 & 97 \\
\hline Total & 365 & 100,0 & 35.300 \\
\hline
\end{tabular}

* Estimación por extrapolación de los resultados del estudio al conjunto de población mayor de 65 años que vive sola en Gipuzkoa. Fuente: Fundación Hurkoa, 2019.

\section{Factor 6. Bienestar material}

En cuanto al bienestar material, el sexto factor estudiado, la encuesta recoge información sobre la situación económica de las personas mayores que viven solas y se les pregunta si tienen dificultad para afrontar los gastos generales (alquiler, electricidad, gas, comida, ropa, etc.).

Del estudio se desprende que 8 de cada 10 personas mayores que viven solas indican que no tienen dificultades para afrontar los gastos generales de alquiler, electricidad, gas, comida, ropa, etc. No obstante, un $2 \%$ señala que en el último año ha tenido muchas dificultades para hacer frente a los gastos generales y un $10 \%$ considera haber tenido alguna dificultad. Además, existe un $14 \%$ que afirma no tener calefacción y un $3 \%$ que, aunque la tiene, no la enciende. Por su parte, el $\mathbf{2 1 , 5} \%$ de las personas mayores encuestadas indica que tiene dificultades o siente ansiedad a la hora de realizar gestiones relacionadas con banca, seguros o aspectos legales.

En el factor del bienestar material se califica como frágiles a aquellas personas que presentan más de dos de las siguientes situaciones:

- En los últimos doce meses, ha tenido dificultad para afrontar los gastos generales (alquiler, electricidad, gas, comida, ropa, etc.).

- El nivel de ingresos mensual actual es inferior a 700 euros.

- La vivienda en la que reside es de alquiler o en propiedad con pagos pendientes.

- La vivienda no tiene calefacción o si la tiene no la pone.

- Percepción de haber sufrido abuso económico.

La aplicación de estos criterios indica que la falta de bienestar material es un factor de fragilidad reconocido por el 1,6\% de las personas mayores de 65 años que viven solas de Gipuzkoa. En cifras absolutas, las personas con ausencia de bienestar material se estiman en 580 .

El $30 \%$ de las personas mayores que viven solas plantea una situación de prefragilidad en la dimensión del bienestar material, ya que han indicado afirmativamente en una o dos situaciones de fragilidad del bienestar material. La extrapolación de estos resultados sitúa en 10.000 la cifra de personas mayores 65 años en Gipuzkoa que se encuentran en situación de prefragilidad por debilidad en su bienestar material.

Tabla 12. Bienestar material como factor de riesgo de la fragilidad de las personas mayores de 65 años que viven solas en Gipuzkoa

\begin{tabular}{|l|c|c|c|}
\hline \multirow{2}{*}{$\begin{array}{l}\text { Bienestar } \\
\text { Material }\end{array}$} & \multicolumn{2}{|c|}{ Resultados de la encuesta } & $\begin{array}{c}\text { Estimación } \\
\text { Gipuzkoa* }\end{array}$ \\
\cline { 2 - 4 } & N & $\%$ & N \\
\hline Sin riesgo & 251 & 68,8 & 24.275 \\
\hline Prefrágil & 108 & 29,6 & 10.445 \\
\hline Frágil & 6 & 1,6 & 580 \\
\hline Total & 365 & 100,0 & 35.300 \\
\hline
\end{tabular}

* Estimación por extrapolación de los resultados del estudio a conjunto de población mayor de 65 años que vive sola en Gipuzkoa. Fuente: Fundación Hurkoa, 2019.

\section{Factor 7. Necesidad de vivienda}

El último factor analizado se refiere a la situación en materia de vivienda. Se indaga en aspectos como el régimen de tenencia de la vivienda en la que residen las personas mayores que viven solas, la situación del entorno, del edificio y de la vivienda con relación a la accesibilidad física y la percepción de tener necesidad de cambiar o de rehabilitar su vivienda principal para adecuarse a sus necesidades.

De acuerdo con los resultados obtenidos, prácticamente la totalidad de las personas mayores que conforman un hogar unipersonal de Gipuzkoa cuenta con una vivienda propia y aproximadamente un $7 \%$ necesita reformar o rehabilitar la vivienda. Estas situaciones de necesidad están en muchas ocasiones relacionadas con problemas de accesibilidad del edificio, carencia de ascensor o viviendas no adaptadas a las necesidades de las personas mayores. Cerca de la mitad de las personas entrevistadas confirma que la accesibilidad del edificio en el que viven no es adecuada, y este aspecto incide negativamente en la posibilidad de salir y relacionarse con otras personas.

En el factor de la vivienda, se califica como persona frágil aquella persona que presenta más de dos de las siguientes situaciones:

- El edificio no es accesible hasta el portal (sin escaleras, con rampa).

- El edificio no cuenta con ascensor hasta la planta.

- La vivienda no es accesible por dentro (no tiene el baño adaptado).

- Actualmente tiene necesidad de cambiar o de reformar la vivienda.

La aplicación de estos criterios indica que el 11,5\% de las personas mayores de 65 años que viven solas de 
Gipuzkoa se encuentra en una situación de fragilidad por motivos de vivienda. En cifras poblacionales, se estima que unas 4.000 personas mayores cuentan con unas condiciones de vivienda no adecuadas. Además, el $52 \%$ reconoce que tiene una o dos de los indicadores de vivienda recogidos, es decir, se encuentra en una situación de prefragilidad por cuestiones relativas a la vivienda. Extrapolando a cifras absolutas, más de 18.000 personas mayores de 65 años se encuentran en esta situación.
Tabla 13. La vivienda como factor de riesgo de la fragilidad de las personas mayores de 65 años que viven solas en Gipuzkoa

\begin{tabular}{|l|c|c|c|}
\hline \multirow{2}{*}{ Vivienda } & \multicolumn{2}{|c|}{ Resultados de la encuesta } & $\begin{array}{c}\text { Estimación } \\
\text { Gipuzkoa* }\end{array}$ \\
\cline { 2 - 4 } & $\mathbf{N}$ & $\%$ & $\mathbf{N}$ \\
\hline Sin riesgo & 133 & 36,4 & 12.863 \\
\hline Prefrágil & 190 & 52,1 & 18.375 \\
\hline Frágil & 42 & 11,5 & 4.062 \\
\hline Total & 365 & $\mathbf{1 0 0 , 0}$ & $\mathbf{3 5 . 3 0 0}$ \\
\hline
\end{tabular}

* Estimación por extrapolación de los resultados del estudio al conjunto de población mayor de 65 años que vive sola en Gipuzkoa. Fuente: Fundación Hurkoa, 2019.

Tabla 14. Principales indicadores de la fragilidad de las personas mayores de 65 años de Gipuzkoa

\begin{tabular}{|c|c|c|c|c|}
\hline DIMENSIÓN & $\begin{array}{l}\text { FACTOR DE } \\
\text { FRAGILIDAD }\end{array}$ & \multicolumn{2}{|l|}{ INDICADOR DE FRAGILIDAD } & $\begin{array}{l}\text { ESTIMACIÓN } \\
\text { GIPUZKOA }\end{array}$ \\
\hline \multirow{5}{*}{ 1. Salud } & 1 & Considera que su estado de salud en general es... & Muy malo/Malo & 4,90 \\
\hline & 2 & ¿Ha estado hospitalizado? & $\begin{array}{l}\text { Sí, en más de una } \\
\text { ocasión }\end{array}$ & 2,50 \\
\hline & 3 & $\begin{array}{l}\text { ¿Tiene con frecuencia problemas de salud que le impiden } \\
\text { valerse por sí mismo? }\end{array}$ & Sí & 9,60 \\
\hline & 4 & ¿Hay más de dos días a la semana que no come caliente? & Sí & 6,60 \\
\hline & 5 & $\begin{array}{l}\text { En su opinión, ¿tiene dificultades para organizar y } \\
\text { gestionar los medicamentos que debe tomar? }\end{array}$ & $\begin{array}{l}\text { Sí, alguna dificultad/ } \\
\text { Sí, bastante } \\
\text { dificultad }\end{array}$ & 1,90 \\
\hline \multirow{7}{*}{$\begin{array}{l}\text { 2. Capacidad para } \\
\text { realizar las actividades } \\
\text { de la vida diaria }\end{array}$} & 6 & $\begin{array}{l}\text { En general, ¿cómo califica su capacidad para realizar las } \\
\text { actividades y labores habituales, de la vida diaria? }\end{array}$ & Muy malo/Malo & 5,50 \\
\hline & 7 & Grado de autonomía: lavarse (bañarse) & $\begin{array}{l}\text { Con dificultad } \\
\text { (dependiente) }\end{array}$ & 1,90 \\
\hline & 8 & Grado de autonomía: vestirse y desvestirse & $\begin{array}{l}\text { Con dificultad } \\
\text { (dependiente) }\end{array}$ & 1,10 \\
\hline & 9 & Grado de autonomía: moverse dentro de casa (50 metros) & $\begin{array}{l}\text { Con dificultad } \\
\text { (dependiente) }\end{array}$ & 0,80 \\
\hline & 10 & $\begin{array}{l}\text { Grado de autonomía: / moverse fuera de casa (Caminar } \\
400 \text { metros sin fatigarse) }\end{array}$ & $\begin{array}{l}\text { Con dificultad } \\
\text { (dependiente) }\end{array}$ & 4,70 \\
\hline & 11 & Grado de autonomía: tareas domésticas (limpieza, comida) & $\begin{array}{l}\text { Con dificultad } \\
\text { (dependiente) }\end{array}$ & 10,10 \\
\hline & 12 & $\begin{array}{l}\text { Grado de autonomía: realizar gestiones económicas o } \\
\text { legales }\end{array}$ & $\begin{array}{l}\text { Con dificultad } \\
\text { (dependiente) }\end{array}$ & 10,70 \\
\hline \multirow{4}{*}{ 3. Red de apoyo social } & 13 & ¿Dispone de apoyo familiar suficiente? & No & 5,80 \\
\hline & 14 & ¿Necesita que le ayude alguien a menudo? & Sí & 11,80 \\
\hline & 15 & ¿Tiene relación? Amigos/as & $\begin{array}{l}\text { No tiene ese vínculo/ } \\
\text { Tiene esa red social, } \\
\text { pero no se relaciona } \\
\text { con ellos }\end{array}$ & 15,10 \\
\hline & 16 & Señale si conoce los servicios sociales & No & 12,10 \\
\hline \multirow{3}{*}{$\begin{array}{l}\text { 4. Participación social } \\
\text { y comunitaria }\end{array}$} & 21 & Frecuencia: salir de casa & $\begin{array}{l}\text { Mensualmente o } \\
\text { menos }\end{array}$ & 4,90 \\
\hline & 22 & $\begin{array}{l}\text { Frecuencia: actividades de participación social (hacer } \\
\text { recados, ir al bar o a la cafetería, ir al hogar del jubilado) }\end{array}$ & No voy nunca & 1,60 \\
\hline & 23 & $\begin{array}{l}\text { Frecuencia: Actividades de participación comunitaria (Salir } \\
\text { a pasear, hacer deporte, ir a actos deportivos o culturales, } \\
\text { cursos de formación, vacaciones o voluntariado) }\end{array}$ & No voy nunca & 2,20 \\
\hline \multirow{4}{*}{ 5. Bienestar emocional } & 17 & Soledad & Sí, a menudo & 17,30 \\
\hline & 18 & Inseguridad en la vivienda (miedo a que le roben, etc.) & Sí, a menudo & 5,20 \\
\hline & 19 & Inseguridad en el entorno (barrio) & Sí, a menudo & 0,80 \\
\hline & 20 & Maltrato físico o psicológico & $\begin{array}{l}\text { Sí, a menudo/Sí, } \\
\text { alguna vez }\end{array}$ & 0,80 \\
\hline
\end{tabular}




\begin{tabular}{|c|c|c|c|c|}
\hline DIMENSIÓN & $\begin{array}{l}\text { FACTOR DE } \\
\text { FRAGILIDAD }\end{array}$ & \multicolumn{2}{|l|}{ INDICADOR DE FRAGILIDAD } & $\begin{array}{l}\text { ESTIMACIÓN } \\
\text { GIPUZKOA } \\
(\%)\end{array}$ \\
\hline \multirow{5}{*}{ 6. Bienestar material } & 24 & $\begin{array}{l}\text { En los últimos doce meses, ¿ha tenido dificultad para } \\
\text { afrontar los gastos generales (alquiler, electricidad, gas, } \\
\text { comida, ropa,...? }\end{array}$ & Mucha dificultad & 2,20 \\
\hline & 25 & $\begin{array}{l}\text { Nos podría indicar cuál es su nivel de ingresos mensual } \\
\text { actual }\end{array}$ & $\begin{array}{l}\text { Menos de } 700 \text { euros } \\
\text { al mes }\end{array}$ & 15,60 \\
\hline & 26 & La vivienda en la que reside es: & $\begin{array}{l}\text { Una vivienda propia } \\
\text { en proceso de pago/ } \\
\text { Una vivienda de } \\
\text { alquiler }\end{array}$ & 4,70 \\
\hline & 27 & ¿La vivienda tiene calefacción? & $\begin{array}{l}\text { No, no tengo/Sí, } \\
\text { tengo calefacción } \\
\text { pero no la pongo }\end{array}$ & 16,70 \\
\hline & 28 & Abuso económico & $\begin{array}{l}\text { Sí, a menudo /Sí, } \\
\text { alguna vez }\end{array}$ & 1,10 \\
\hline \multirow{4}{*}{$\begin{array}{l}\text { 7. Necesidad de } \\
\text { vivienda }\end{array}$} & 29 & $\begin{array}{l}\text { El edificio es accesible hasta el portal (sin escaleras, con } \\
\text { rampa) }\end{array}$ & No & 41,40 \\
\hline & 30 & Cuenta con ascensor hasta la planta & /No & 33,20 \\
\hline & 31 & $\begin{array}{l}\text { La vivienda es accesible por dentro (tiene el baño } \\
\text { adaptado) }\end{array}$ & No & 29,30 \\
\hline & 32 & $\begin{array}{l}\text { ¿Actualmente tiene necesidad de cambiar o de reformar la } \\
\text { vivienda? }\end{array}$ & $\begin{array}{l}\text { Necesito cambiar de } \\
\text { vivienda / Necesito } \\
\text { reformar o rehabilitar } \\
\text { la vivienda }\end{array}$ & 7,10 \\
\hline
\end{tabular}

* Estimación por extrapolación de los resultados del estudio al conjunto de población mayor de 65 años que vive sola en Gipuzkoa.

Fuente: Fundación Hurkoa, 2019.

\section{Algunos aspectos a tener en cuenta para concluir}

El estudio realizado pone de manifiesto unos niveles de fragilidad que se podrían calificar de moderados entre las personas mayores que viven solas en Gipuzkoa. Los resultados obtenidos en los tres municipios incluidos en la encuesta señalan que solo un $1,4 \%$ de estas personas viven situaciones graves de fragilidad (en torno a 500 personas, en cifras extrapoladas), aunque un $12 \%$ presenta una fragilidad moderada (más de 4.000 personas).

Los factores relacionados con la soledad y el aislamiento social resultan los más problemáticos, seguidos de los relativos a los problemas de salud. En una posición intermedia se encuentran los factores de fragilidad relacionados con la capacidad para realizar las actividades de la vida diaria y con la calidad de vida en general, mientras que la mejor situación corresponde a los factores económicos y a la disposición de apoyos.

Estos resultados ponen de relieve que el contexto de fragilidad de las personas mayores requiere del reconocimiento de la convergencia y combinación que surge entre diversos factores, tales como el estado de salud, la capacidad para realizar las actividades diarias, la red de apoyo social, el bienestar emocional, la participación social y comunitaria, el bienestar material y las condiciones y accesibilidad de la vivienda. El abordaje de estas situaciones de fragilidad requiere un enfoque multidimensional, lo que pone en valor la necesaria colaboración entre los y las profesionales del ámbito social y de la salud, así como la participación del conjunto de agentes de la comunidad.

Si se tienen en cuenta las dimensiones arriba indicadas, es propio concluir que resulta pertinente desarrollar un trabajo desde la proximidad, concepto que hace referencia a la idea de proximidad activa, entendida como la práctica de acercarse al lugar donde esté la persona, con una actitud proactiva para conocer al sujeto y su entorno. Este concepto incluye también, la idea de proximidad construida, aspecto que subraya la importancia de crear y construir un vínculo de cercanía y enganche entre la persona mayor que está en la situación de fragilidad y el profesional (Aguilar y Llobet, 2013). Por otro lado, la fragilidad requiere del acompañamiento, entendido como método de intervención profesional orientado a atender las necesidades de apoyo social para lograr la participación de la persona en su entorno comunitario y mejorar el grado de satisfacción de las necesidades sociales, instrumentales y relacionales (Red Navarra de Lucha contra la Pobreza y la Exclusión Social, 2011).

Ante estas consideraciones, es importante que el modelo de atención de las personas mayores en situación de fragilidad, quede vinculado, por una parte, a la intervención individual (gestión del caso) con el objetivo de cubrir las necesidades y mejorar la calidad de vida de esta; $y$, por otra, a la intervención comunitaria, haciendo una labor de sensibilización con los agentes sociales, para que apoyen la detección de las situaciones de fragilidad y a su vez faciliten la proximidad de estas personas a los recursos del entorno. 
Esta doble dimensión, posibilita a su vez concretar los apoyos que se precisan para el trabajo y desarrollo de intervenciones integrales y coordinadas, atendiendo a las distintas necesidades identificadas en torno a tres cuestiones clave:

- Apoyo instrumental, orientado a la realización de trámites vinculados a los recursos sociales y económicos, organización del cuidado personal, orientación en la adecuación de los hábitos, asesoramiento en diversas gestiones como la lectura de facturas y otros documentos, etc.
- Apoyo social y sentido de pertenencia, que busca facilitar el acceso a los recursos del entorno comunitario, facilitar el vínculo con la red social y comunitaria, etc.

- Apoyo emocional, necesario para fomentar la seguridad y empoderamiento de la persona mayor, facilitando las relaciones significativas. 


\section{Referencias bibliográficas}

AGUILAR, M. y LLOBET, M. (2013): “Servicios sociales: integralidad, acompañamiento, proximidad, incorporación", Encuentros de expertos en Inclusión social en Mérida IntegrACTIVA, Fundación Luis Vives.

FUNDACIÓN HURKOA (2018): Informe del Proyecto de Fragilidad, Vitoria-Gasteiz, Departamento de Empleo y Políticas Sociales del Gobierno Vasco, «https://www.euskadi.eus/contenidos/ informacion/publicaciones_ss/es_publica/ adjuntos/INFORME\%20FRAGILIDAD2018_ES_ WEB.pdf .

- (2019): La fragilidad de las personas mayores en Gipuzkoa, Fundación Hurkoa, <https://www. hurkoa.eus/sites/default/files/fragilidad_ personas_mayores.pdfhttps://www.hurkoa. eus/sites/default/files/fragilidad_personas_ mayores.pdf〉.

JÁUREGUI, J.R. y RUBIN, R. (2012): “Fragilidad en el adulto mayor", Revista del Hospital Italiano de Buenos Aires, vol. 32, no 3, pp. 110-115, <https://www.hospitalitaliano.org.ar/ multimedia/archivos/noticias_attachs/47/ documentos/12815_110-115_Hl3-2_Revision_ Jauregui\%2oRubin.pdf〉.

RED NAVARRA DE LUCHA CONTRA LA POBREZA Y LA EXCLUSIÓN SOCIAL (2011): El acompañamiento como método de intervención en los procesos de inclusión: nuevas reflexiones, Red Navarra de Lucha contra la Pobreza y la Exclusión Social. 